\title{
Perspective
}

PERSPECTIVE Actualité en histoire de l'art

1 | 2008

Antiquité/Moyen Âge

\section{L'histoire des collections : au-delà du temps des pionniers}

Krzysztof Pomian

\section{OpenEdition}

1 Journals

Édition électronique

URL : http://journals.openedition.org/perspective/3485

DOI : 10.4000/perspective.3485

ISSN : 2269-7721

Éditeur

Institut national d'histoire de l'art

\section{Édition imprimée}

Date de publication : 31 mars 2008

Pagination : 5-7

ISSN : 1777-7852

\section{Référence électronique}

Krzysztof Pomian, "L'histoire des collections : au-delà du temps des pionniers », Perspective [En ligne], 1 | 2008, mis en ligne le 31 mars 2018, consulté le 01 octobre 2020. URL : http:// journals.openedition.org/perspective/3485; DOI : https://doi.org/10.4000/perspective.3485 
Krzysztof Pomian

\section{L'histoire des collections : au-delà du temps des pionniers}

r éservée naguère à l'érudition locale, l'histoire des collections est devenue au cours des trois dernières décennies une branche à part entière de l'histoire culturelle. t out en étant étudiées pour elles-mêmes comme un phénomène suigeneris, les collections le sont en effet aussi parce qu'elles permettent d'appréhender les attitudes cognitives et esthétiques, les pratiques intellectuelles, les relations sociales, les représentations. En tant qu'intermédiaires entre l'invisible et le visible, les objets qui les composent sont souvent entourés d'une aura de sacralité et investis d'une forte charge symbolique, ce qui leur fait attribuer en outre une valeur marchande. À ces différents titres, les collections intéressent l'histoire de l'art et l'histoire des sciences, l'histoire des institutions et des politiques culturelles, l'histoire religieuse, l'histoire politique et même l'histoire économique et sociale.

l a collection est un fait anthropologique coextensif à l'homo sapiens et présent sous plusieurs formes, notamment sous celle des trésors des temples et des palais dont procèdent les dépôts funéraires, sous celle des collections particulières, sous celle des musées. Mais l'histoire des collections est restée longtemps focalisée sur l'Europe et sur la période comprise entre les $\mathrm{xv}^{\mathrm{e}}$ et $\mathrm{xvii}^{\mathrm{e}}$ siècles. dans ce cadre, elle s'intéressait surtout aux cabinets des curiosités et aux galeries d'art. Ce n'est que depuis quelques années qu'elle s'en éloigne tant dans l'espace que dans le temps. dans l'espace : qu'il suffise de mentionner les recherches sur les collections en Chine, seule civilisation, avec l'ancienne r ome et la chrétienté latine à partir du xive siècle, à avoir inventé la collection particulière. dans le temps, car elle se tourne maintenant vers d'autres époques.

Et d'abord, vers l'a ntiquité. Un travail récent consacré aux manières qu'avaient les Grecs de reconstituer leur passé mythique projette ainsi une nouvelle lumière sur les collections qui entre autres s'amassaient dans leurs temples, de même qu'une recherche traite des chasseurs des fossiles, cependant que l'étude à partir des vestiges architecturaux des trésors de certaines cités dans de grands sanctuaires permet de mieux comprendre leurs rôles tant dans l'économie que dans la rivalité pour le prestige ou la renommée. Mais c'est surtout l'histoire des collections romaines qui, au cours de dernières années, a été enrichie par les recherches sur le butin de guerre et les usages qu'en faisaient les généraux victorieux, sur les masques des ancêtres et leur place dans la vie de l'aristocratie, sur le projet encyclopédique de Pline l'a ncien, auteur dont il est inutile de souligner l'importance essentielle pour notre sujet, sur la sculpture, la peinture et la vie sociale de cette dernière à r ome même et dans les villes du golfe de Naples, sur le trésor impérial, enfin, à la jointure de l'économie, du pouvoir et du sacré. 
Présents partout où il existait des pouvoirs sacerdotaux ou des monarchies sacrées, les trésors de différentes époques bénéficient maintenant d'un regain d'intérêt. Mais on les étudie principalement, à ce qu'il semble, sous la forme qu'ils ont reçue dans le Moyen Âge latin. d es inventaires en ont été publiés en renouant le fil avec les publications similaires du xix ${ }^{\mathrm{e}}$ siècle, cependant qu'on mettait en lumière des images qui les représentaient et des descriptions verbales dont ils furent l'objet, et qu'on a analysé leur composition et leurs rôles sociaux et culturels. Semblablement, de nombreux travaux dans différents pays portent sur des reliques : sur les significations dont elles étaient investies, sur les usages qu'on en faisait, sur leur importance cérémonielle et, partant, politique, sur les vols, les dons et les échanges dans lesquels elles étaient engagées, sur les sentiments qu'elles éveillaient et les comportements collectifs qu'elles suscitaient. À la différence des chercheurs qui étudient les trésors, les auteurs des travaux sur les reliques ne semblent pas en général avoir conscience d'appartenir à l'histoire des collections. C'est pourtant le cas.

$\mathrm{d} u$ côté du Moyen Âge, les trésors et les reliques. du côté de la période moderne, les musées et les monuments. En effet, l'histoire des musées s'est enrichie récemment d'un grand nombre de publications qui l'ont littéralement renouvelée. Nous connaissons aujourd'hui comme jamais auparavant l'histoire des musées en Espagne et, beaucoup mieux qu'avant, l'histoire des musées dans presque tous les pays européens, notamment en a llemagne. Nous disposons des biographies d'un certain nombre de grandes figures tels Vivant denon, von Bode, o skar Müller ; de monographies de plusieurs établissements - pour certains d'importance mondiale, pour d'autres de portée locale seulement, ce qui n'enlève rien à leur intérêt, bien au contraire; d'études consacrées à différents types de musées, au musée d'art en premier lieu, mais aussi au musée d'ethnographie, au musée d'histoire, aux musées des sciences et des techniques. Un trait marquant de l'histoire des musées telle qu'elle se pratique aujourd'hui, c'est qu'elle ne s'enferme pas en Europe. En fait, c'est l'histoire des musées aux états-Unis qui est probablement la mieux connue. Mais les dernières années ont apporté aussi un lot de travaux sur les musées en turquie, en égypte, en a frique noire, en a mérique du Sud et dans d'autres sociétés où ils ont été amenés par les Européens et qui les ont assimilés à des degrés fort différents en fonction des traditions locales et des conjonctures économiques et politiques.

Cet élargissement du champ ne signifie pas que la période comprise entre le $\mathrm{xv}^{\mathrm{e}}$ et le xviii ${ }^{\text {e }}$ siècle en Europe ait été abandonnée. il faut noter plus spécialement l'essor des recherches dans les pays où il restait beaucoup à faire, comme en Espagne, ou carrément laissés en friche il y a peu encore : en Pologne, en r ussie. o n mentionnera, pour leur qualité exceptionnelle et leur portée, les travaux réalisés conjointement par les historiens néerlandais et russes autour des collections de Pierre le Grand.

l a littérature sur les collections est foisonnante au point où il est à craindre que personne ne la maîtrise plus dans son intégralité. Mais même des lectures partielles complétées par les comptes rendus dans l'irremplaçable Journal of the History of Collections permettent de dire que le temps commence à être mûr pour passer au niveau supérieur. Ce dont nous avons besoin maintenant, c'est de tentatives de comparaison entre les différentes civilisations, là où cela peut être pertinent; cela concerne notamment les trésors et les reliques, mais il serait aussi d'un grand intérêt de pouvoir comparer 
l'histoire des collections particulières en o ccident et en Chine. Nous avons besoin aussi de sortir du cadre national et pas seulement de comparer différents pays européens et, en outre, d'essayer de saisir les collections à l'échelle de l'Europe entre le xv ${ }^{\mathrm{e}}$ et le xix ${ }^{\mathrm{e}}$ siècle : entre le moment où elles commencent à se constituer et celui de leur dispersion, suivie dans beaucoup de cas de l'entrée des objets dans les musées en Europe même, aux é tats-Unis ou ailleurs.

Pour ce qui est de l'histoire des musées, on aurait besoin de la mettre au service d'une histoire de l'unification artistique et culturelle de l'Europe. l a propagation des musées, en effet, est inséparable de la circulation des antiquités grecques et romaines, des originaux mêmes ou des copies en plâtre, et des reproductions dans des matériaux et des formats divers. Elle est inséparable aussi de la circulation des ouvres modernes qui, pendant longtemps, transposent et réactualisent des modèles antiques. d es légions romaines se sont arrêtées sur la ligne du $\mathrm{r}$ hin et du danube. Et c'est mille ans après la chute de l'Empire romain en o ccident que des ouvres d'art romaines et grecques ont traversé le ci-devant limes en montant du sud vers le nord, en exerçant une influence sur l'art et plus généralement sur les paysages des contrées qui jadis faisaient partie du barbaricum. Qu'il suffise de penser à l'empreinte laissée par le néoclassicisme en a llemagne, en Grande-Bretagne, au danemark, en Pologne, en r ussie ou en Suède.

depuis le temps des pionniers - Francis Haskell, o liver impey, a dalgisa l ugli et a ntoine Schnapper, pour ne mentionner que les morts -, l'histoire des collections a parcouru un long chemin. Portée par le profond changement qui a détrôné l'histoire économique et sociale de son rôle de rectrice de l'ensemble des disciplines historiques pour conférer ce rôle à une histoire culturelle rendue sensible à la dimension spatiale et matérielle des phénomènes qu'elle étudie, elle a changé de statut et de portée dans le cadre de l'histoire de l'art, suite au déplacement de l'intérêt de celle-ci de la création vers la réception. Elle est entrée désormais dans l'âge adulte, ce qui rend d'autant plus impératif un profond renouvellement de ses questionnaires et un changement de ses perspectives. 